Check for updates

Cite this: RSC Adv., 2019, 9, 14051

Received 31st March 2019

Accepted 1st May 2019

DOI: $10.1039 / c 9 r a 02430 a$

rsc.li/rsc-advances

\section{Evaluation of non-targeting, C- or N-pH (low) insertion peptide modified superparamagnetic iron oxide nanoclusters for selective MRI of liver tumors and their potential toxicity in cirrhosis $\uparrow$}

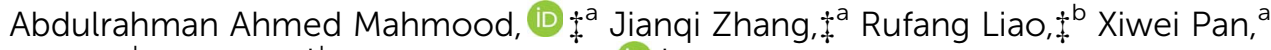

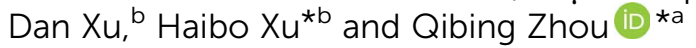

Superparamagnetic iron oxide nanoclusters (SPIONs) modified with $\mathrm{pH}$ (low) insertion peptide (pHLIP) could be advantageous for magnetic resonance imaging (MRI) diagnosis of liver tumors at the early stage due to their unique responsiveness to the tumor acidic microenvironment when tumor markers are unknown. However, many critical aspects including the effectiveness of selective MRI in liver tumors, types of delivery and the potential safety profile in cirrhosis need to be fully evaluated. In this study, we report the evaluation of non-targeting, $\mathrm{C}$ - or N-pHLIP modified SPIONs as the contrast agent for selective MRI of liver tumors and their potential toxicity profile in cirrhosis. It was found that N-pHLIP modified SPIONs did not result in the loss of liver tumor in the T2-weight MRI but provided additional dynamic details of tumor structures that would enhance the diagnosis of liver tumors at a small size below $8 \mathrm{~mm}$. In addition, an enhanced safety profile was found for N-pHLIP modified SPIONs with almost fully recoverable impact in cirrhosis. In contrast, the poly-D-lysine assembled SPIONs and Cterminus linked pHLIP SPIONs had non-tumor specific MRI contrast enhancement and potential safety risks in cirrhosis due to the iron overload post injection. All these results implied the promising potential of $\mathrm{N}$-terminus linked pHLIP SPIONs as an MRI contrast agent for the diagnosis of liver tumors.
\end{abstract}

\section{Introduction}

Liver cancer has been one of the major cancer burdens with increasing mortalities over recent years from 695000 in 2008 to 781631 in 2013 according to reports by the global cancer observatory. ${ }^{1}$ On the basis of the Barcelona clinic liver cancer staging system, the early diagnosis and treatment of liver cancer at stage 0 (single tumor $<2 \mathrm{~cm}$ ) could effectively increase the 5 year survival rate up to $70 \% .^{2,3}$ Magnetic resonance imaging (MRI) has been an effective non-invasive diagnostic method for liver tumors, yet the challenge remains in the early liver tumor diagnosis due to limits in distinguishing them from liver lesions such as cirrhosis., ${ }^{3,4}$ Superparamagnetic iron oxide

\footnotetext{
${ }^{a}$ Department of Nanomedicine \& Biopharmaceuticals, College of Life Science and Technology, National Engineering Research Center for Nanomedicine, Huazhong University of Science and Technology, Wuhan 430074, Hubei, China. E-mail: qibingzhou@hust.edu.cn

${ }^{b}$ Department of Radiology, Zhongnan Hospital of Wuhan University, Wuhan 430071, Hubei, China. E-mail: xuhaibo1120@hotmail.com

$\dagger$ Electronic supplementary information (ESI) available: Synthetic procedures for the of hydroxyethyl starch coated SPIO; Fig. S1: TEM image of the synthesized hydroxyethyl starch coated SPIO; Fig. S2: stability of assembled SPION in $10 \%$ serum over time. See DOI: 10.1039/c9ra02430a

\$ These authors contributed equally.
}

particles (SPIOs) have been used as an MRI contrast enhancement agent to improve the diagnostic accuracy of liver tumors in cirrhosis. ${ }^{5-8}$ In addition, SPIO has also been used to assess stages of cirrhosis and non-alcoholic steatohepatitis as well as the effectiveness of treatment of non-alcoholic fatty liver disease. ${ }^{9-13}$ Furthermore, SPIO has been widely used in diagnosis and therapy of other cancers such as prostate cancer diagnosis and drug delivery. ${ }^{\mathbf{1 4 - 1 9}}$ On the other hand, the potential cytotoxicity of SPIO has recently been considered as a major safety concern, ${ }^{\mathbf{1 9 - 2 1}}$ especially in chronic liver diseases due to SPIO induced iron overload resulting in increased risks of steatohepatitis and cirrhosis progression and liver lesions in obesity. ${ }^{22-26}$ One way to enhance the safety profile of SPIO was to utilize the target-specific delivery strategy such as cRGD ligand or $\mathrm{pH}$ (low) insertion peptide (pHLIP). ${ }^{27-30}$ pHLIP has been successfully utilized to target tumor-specific acidic microenvironment due to its unique conformational change under acidic conditions to $\alpha$-helical membrane insertion mode. ${ }^{31-33}$ It was specially advantageous for early tumor diagnosis because the molecular markers and subtypes of liver tumors in most cases are unknown at early stage. Furthermore, pHLIP is capable to achieve dual modes of cargo delivery to tumor cells through peptide modification at either $\mathrm{N}$ - or C-terminus. ${ }^{33,34}$ We recently reported that pHLIP linked SPIO nanoclusters (SPION) could be 
an effective contrast agent in the MRI of subcutaneous tumor models. ${ }^{30}$ However, many critical aspects remain unclear in the liver tumor diagnosis. For example, a critical concern is whether the accumulation of targeting SPION in liver tumor would result in the full disappearance of the tumor in T2-weighted MRI due to the concurrent uptake of SPION by the normal liver Kupffer cells. Another one is what significant advantages pHLIP modified SPIONs could have over non-targeting SPIO for the MRI of the orthotopic liver tumor as well as the difference between the C- and N-terminus pHLIP delivery of SPION. Further question is how the targeting SPIONs would have any improvement in the safety profile in cirrhosis due to the iron overload. In this study, we report the evaluation of the effectiveness of non-targeting, Cor N-pHLIP modified SPION for selective MRI of liver tumor and their potential toxicity profile in cirrhosis as the potential MRI contrast agent.

\section{Material and methods}

All chemicals were obtained from Sigma-Aldrich (USA) and Sinopharm Chemical Reagent Co., Ltd. (China) unless specified otherwise. The hydroxyethyl starch coated SPIO was synthesized as reported, ${ }^{35}$ and the detailed steps were described in the ESI. $\dagger$ Murine liver cancer cell line H22 were obtained from Shanghai Institute of Life Science Cell Culture Center (China) and maintained via passages intraperitoneally in BALB/c mice.

\subsection{Synthesis of pHLIP modified poly-D-lysine polymers}

C- and N-terminal inserted cysteine pHLIP peptides were commercially synthesized by GenScript (NJ, USA) with certified analysis (protein sequences shown in Fig. 1a). Poly-D-lysine (PDL, 30-70 kDa) in phosphate buffered saline (PBS, $1 \mathrm{mg}$ $\left.\mathrm{mL}^{-1}, 100 \mu \mathrm{L}\right)$ was first mixed with maleimide-(PEG) $)_{24}$-succinimidyl ester (Thermo Scientific Pierce, USA) in DMSO (10 $\mu \mathrm{g}$ $\mu \mathrm{L}^{-1}, 20 \mu \mathrm{L}$ ) at room temperature. After $1 \mathrm{~h}$, the PEG-maleimide modified PDL was purified via centrifugation with a Zeba spin desalting column (7 kDa cutoff, Thermo Fisher Scientific, USA). The resulting filtrate was then mixed with pHLIP peptide solutions $\left(2 \mu \mathrm{g} \mu \mathrm{L}^{-1}, 100 \mu \mathrm{L}\right.$ in PBS). The desired pHLIP modified PDLs were obtained through centrifugation with the Zeba spin desalting column. The UV absorbance spectra of the resulting modified polymers were obtained with a T9 UV-Vis spectrometer (Persee Analytics Inc., China) in PBS. The fluorescence emission spectra were recorded with an F-4500 fluorescence spectrometer (Hitachi, Japan) using the excitation wavelength at $280 \mathrm{~nm}$. The circular dichroism (CD) spectra were obtained with a J-810 CD spectrometer (JASCO Inc., USA) at $25^{\circ} \mathrm{C}$ in PBS at $\mathrm{pH}$ 4.0 and 8.0 containing $1.0 \mathrm{mg} \mathrm{mL}{ }^{-1}$ lipids including 1,2-distearoyl-sn-glycero-3-phosphocholine, 1,2-dioleoyl-sn-glycero-3phosphocholine and cholesterol at a weight ratio of $2: 1: 1$.

\subsection{Assembly of SPIO nanoclusters}

Three types of SPIO nanoclusters were prepared with PDL, Cand N-terminus linked pHLIP PDL polymers with the hydrodynamic diameter size around $70 \mathrm{~nm}$ as PDL-SPION, C-pHLIPSPION and N-pHLIP-SPION, respectively. Typically, the poly-

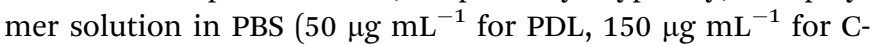

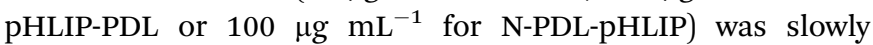
added to an SPIO solution (10 $\mathrm{mg} \mathrm{mL}^{-1}$ in PBS at 8.0) at $1: 1$ volume ratio under sonication and then equilibrated at room

(a)

pH Low Insertion peptide (pHLIP)

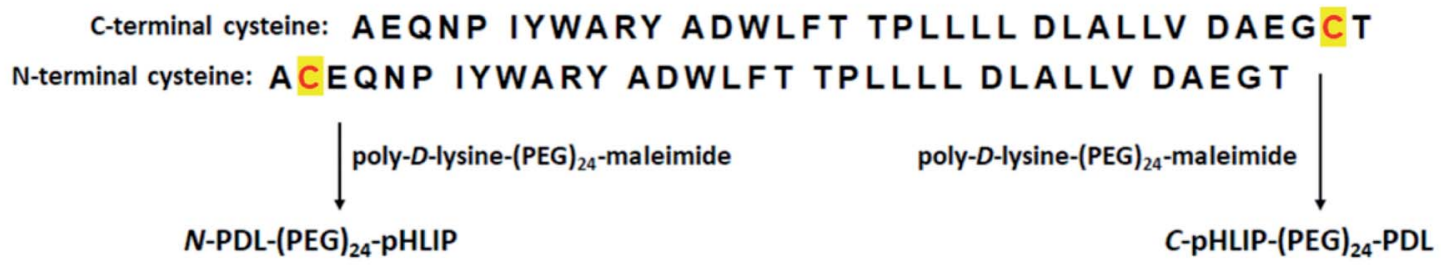

(b)

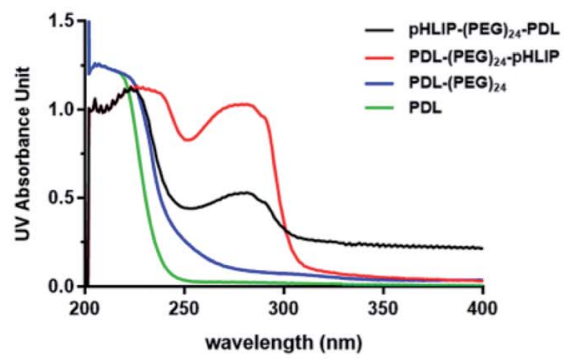

(c)

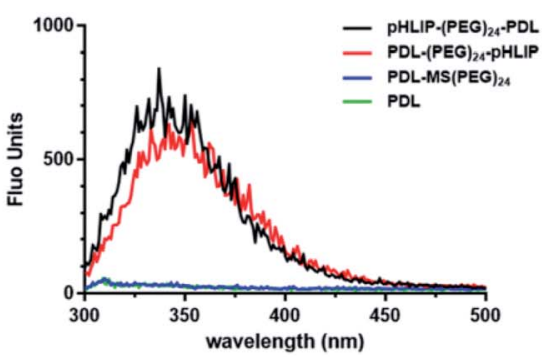

(d)

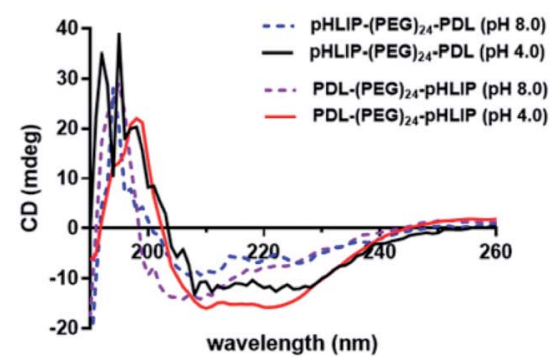

Fig. 1 Synthesis of pHLIP modified PDL polymers and the chemical physical properties. (a) Protein sequences of pHLIP peptides with cysteine inserted at the C-terminus or N-terminus and the synthetic steps; (b) UV spectra of pHLIP modified PDL polymers; (c) fluorescence emission spectra of pHLIP modified PDL polymer (excitation at $280 \mathrm{~nm}$ ); (d) CD spectra of pHLIP-modified PDL polymers in PBS solutions at pH 4.0 and pH 8.0 containing $1 \mathrm{mg} \mathrm{mL}^{-1}$ lipids. 
temperature for $20 \mathrm{~h}$ to afford the desired nanoclusters. The hydrodynamic size and zeta-potential of the nanoclusters were determined with a Nano-ZS90 particle analyzer (Malvern, United Kingdom) in PBS. The transmission electron microscopic images were obtained with a Hitachi HT-7700 transmission electron microscope (Tokyo, Japan) using uranyl acetate staining. The stability of SPION was assessed in PBS containing $10 \%$ FBS over $24 \mathrm{~h}$ at $37{ }^{\circ} \mathrm{C}$ with the Nano-ZS90 particle analyzer. The relaxivity $\left(R_{2}\right)$ of assembled nanoclusters was obtained as the slope of a series of SPION concentrations over the 1/T2 that was determined with a clinical Magnetom Trio Tim MRI spectrometer (3.0 Tesla, Siemens Prisma, Germany) as reported. ${ }^{35}$

\subsection{Orthotopic liver tumor and cirrhosis mouse models}

All animal procedures were performed in accordance with the Guidelines for Care and Use of Laboratory Animals of the People's Republic of China and approved by the Animal Ethics Committee of Huazhong University of Science and Technology. SFP male BALB/c mice (6-7 weeks of age) were obtained from Beijing HFK Bioscience Co. Ltd., China. For the orthotopic liver tumor model, H22 subcutaneous tumors were first grown at the right flank of BALB/c mice until the tumor size reached $14 \times$ $14 \mathrm{~mm}$ approximately. The tumor tissue was then harvested, and small pieces $(1 \times 1 \mathrm{~mm})$ were inserted in the liver of naive mice through surgical procedure under isoflurane $/ \mathrm{O}_{2}$ anesthesia. Typically, orthotopic liver tumors generally formed in mice over 10-20 days at a successful rate of $30 \%$ to the size of 6 $\times 6 \mathrm{~mm}$ approximately by MRI analysis. For the cirrhotic liver model, diethyl 1,4-dihydro-2,4,6-trimethyl-3,5-pyridinedicarboxylate (Acros Organics, USA) suspended in corn oil (10 $\mathrm{mg} \mathrm{mL}^{-1}$, pharmaceutical grade, Aladdin, China) was fed into mice via gavage at a dose of $0.25 \mathrm{mg} \mathrm{g}^{-1}$ body weight twice a week for four and half weeks. The formation of cirrhosis was confirmed by MRI analysis in week six as significantly enlarged liver at a successful rate of $100 \%$ and directly used for the following studies.

\subsection{In vivo MRI assessment of liver tumors and cirrhosis with SPIONs}

The T2-weighted MRI of the mice were performed with a clinical Magnetom Trio Tim MRI spectrometer (3.0 Tesla, Siemens Prisma, Germany) using optimized sequences of $3000 \mathrm{~ms}$ repetition time, $80 \mathrm{~ms}$ echo time, $1.0 \mathrm{~mm}$ slice thickness, $1.0 \mathrm{~mm}$ slice space thickness and $30 \times 96 \mathrm{~mm}$ field of view. Prior to the SPION injection, MRI was first performed on all mice as the baseline references. SPION solutions including PDL-SPION, C-pHLIP-SPION and N-pHLIP-SPION (100 $\mu \mathrm{L}$ each) were then intravenously injected at $5 \mathrm{mg}$ Fe per $\mathrm{kg}$ body weight ( 3 mice per group). MRI was performed at 0, 2, 4 and $24 \mathrm{~h}$ post iv injection. The resulting MR images were processed with provided Syngo Fastview software (Siemens, Germany).

\subsection{Toxicity profiles of SPION in cirrhosis}

The toxicity profiles of SPION in cirrhosis were assessed through the serum biochemistry tests on day 1, 2, 5, 10 and 15 post the single injection of SPION solutions at $5 \mathrm{mg}$ Fe per $\mathrm{kg}$ body weight ( 5 mice per group). Typically, blood was collected on the specified day, and the obtained sera were assessed with serum biochemistry test kits included levels of alanine aminotransferase (ALT), aspartate aminotransferase (AST), alkaline phosphatase (AKP), cholesterol (CHOL), high-density lipoprotein cholesterol (HDL) and low-density lipoprotein cholesterol (LDL) (Nanjing Jiancheng bioengineering Institute, China). As a comparison, the levels of serum biochemistry in the cirrhosis mice without SPION injection or with injection of nonassembled SPIO were obtained on the same end point (day 15) as the references. In addition, the body weights of mice in all groups were monitored over 15 days post SPION injection. The liver and spleen organ weights were recorded on day 15 post SPION injection. The iron level in the liver tissue was determined similarly as reported..$^{35}$ All the data obtained were analyzed by GraphPad Prism program with one-way ANOVA and Tukey's multiple comparison statistical test.

\section{Results and discussion}

\subsection{Stable SPIO nanoclusters produced through assembly with modified PDL polymers}

Three different types of assembled SPION were assessed in this study, i.e., non-targeting PDL assembled SPION and the C- or Nterminus linked pHLIP PDL assembled SPION. The acidresponsive pHLIP modified PDL polymers were firstly synthesized via the inserted cysteine residue at the $\mathrm{C}$ - or $\mathrm{N}$-terminus of the peptide (sequences shown in Fig. 1a). The conjugation was achieved through the amide formation of the PDL lysine residues with an activated succinimide ester of a PEG crosslinker followed by the thiol addition on the maleimide by pHLIP cysteine (Fig. 1a) as reported. ${ }^{30}$ The incorporation of PEG linkage was to maintain the water solubility of the modified polymers due to the high hydrophobicity of pHLIP peptides. The synthesized C-terminus linked pHLIP PDL (C-pHLIP-PDL) and N-terminus linked pHLIP PDL (N-pHLIP-PDL) polymers had characteristic UV and fluorescence properties as those of pHLIP peptide (Fig. 1b-c). ${ }^{30}$ The amount of pHLIP attachment was estimated to be approximately $5 \%$ of the PDL lysine residues based on the fluorescence intensity of modified polymers. ${ }^{30}$ Moreover, both C- and N-terminus linked pHLIP PDL polymers exhibited conformational changes with more $\alpha$-helix structures at $\mathrm{pH} 4.0$ than those at $\mathrm{pH} 8.0$ (increased negative mdeg around $220 \mathrm{~nm}$ in Fig. 1d).$^{30}$ All these results indicated the successful conjugation of PDL polymers with the pHLIP peptide linked at either $\mathrm{C}$ - or N-terminus.

The assembly of SPIO nanoclusters was then accomplished with PDL and the synthesized PHLIP PDL polymers on the hydroxyethyl starch coated SPIO. The final nanocluster diameter size was optimized to be approximately $70 \mathrm{~nm}$, which was found to be the most effective size for the enhancement in the MRI of subcutaneous tumor models. ${ }^{30}$ The zeta average diameter sizes of the assembled PDL-SPION, and C-pHLIP-SPION and N-pHLIP-SPION were 65.9, 64.8 and $68.1 \mathrm{~nm}$, respectively (Fig. 2a-c). The zeta potentials of all three nanoclusters were similarly in the range of -1.69 to $-1.32 \mathrm{mV}$ in PBS. Moreover, 
(a)

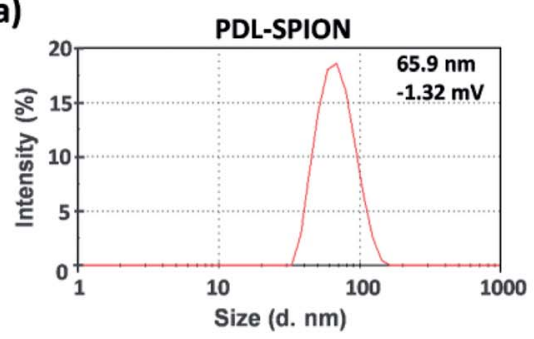

(d)

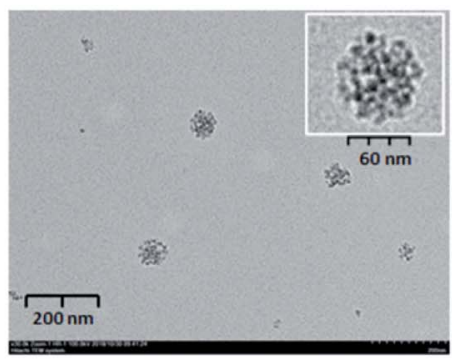

(b)

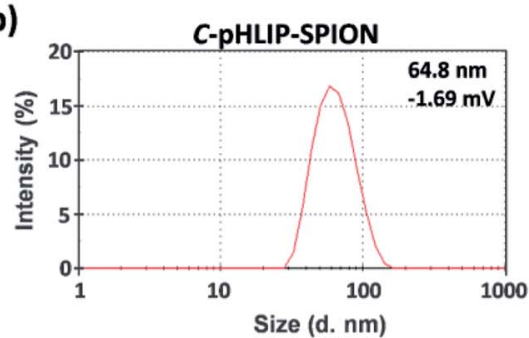

(e)

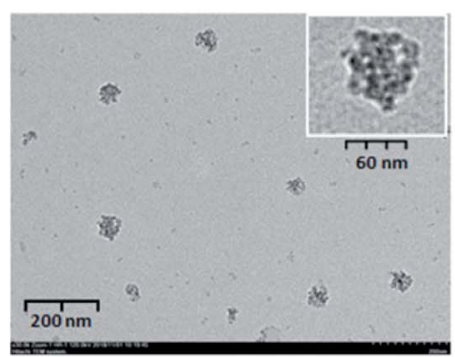

(c)

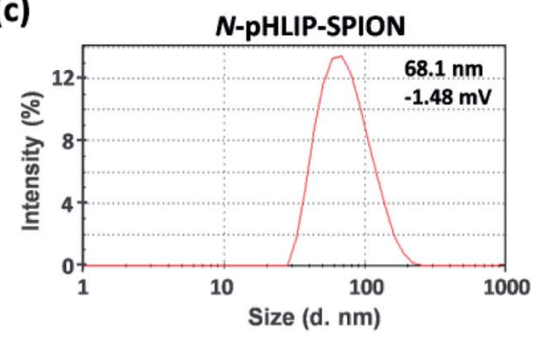

(f)

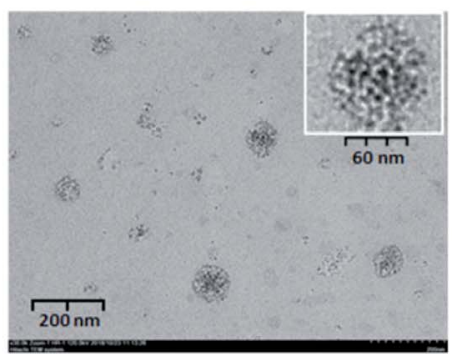

Fig. 2 Physical properties of PDL and pHLIP-modified PDL assembled SPIO nanoclusters. Distribution of nanocluster diameter size as determined by dynamic light scattering for (a) PDL-SPION, (b) C-pHLIP-SPION and (c) N-pHLIP-SPION; TEM images of (d) PDL-SPION, (e) C-pHLIPSPION and (f) N-pHLIP-SPION.

TEM analysis confirmed the assembly of SPIO into the spherical nanoclusters by PDL polymers with consistent diameter size (Fig. 2d-f), which were significantly different from the amorphous distribution of non-assembled hydroxyethyl starch coated SPIO (Fig. S1†). The MRI contrast enhancement capability of assembled SPION were determined as the relaxivity $\left(R_{2}\right)$ of $71.8,72.3$ and $70.7 \mathrm{mM}^{-1} \mathrm{~s}^{-1}$ for PDL-SPION, C-pHLIP-SPION and N-pHLIP-SPION, respectively, which were higher than that of non-assembled SPIO $\left(55.4 \mathrm{mM}^{-1} \mathrm{~s}^{-1}\right)$. Furthermore, the stability of these three assembled SPION was confirmed with no significant change of the zeta average diameter size over $24 \mathrm{~h}$ at $37^{\circ} \mathrm{C}$ in PBS solution (pH 7.4) containing $10 \%$ serum (Fig. S2 $\dagger$ ).

\subsection{Significant MRI enhancement of orthotopic liver tumor with assembled SPION}

The enhancement of MRI contrast by assembled SPION in orthotopic liver tumor was investigated with the tumor of 5$8 \mathrm{~mm}$ size approximately. The T2-weighted MR images were obtained using a clinical 3.0 Tesla MRI instrument with settings optimized for mice study. The resulting representative MR images were shown in Fig. 3 with a scale bar of $5 \mathrm{~mm}$. The slight blurry in MR images was due to the muscle relaxation during the long acquisition time and limits of the instrument. Nevertheless, significant differences were found in MRI post the injection of assembled SPION. Firstly, post the injection of PDLSPION or C-pHLIP-SPION, the MRI signals of the normal liver tissue decreased significantly due to the uptake of SPION by the normal liver Kupffer cells. ${ }^{35}$ On the other hand, the liver tumors remained unchanged post the SPION injection due to the dysfunctional macrophages in tumors. In addition, no significant changes of the tumor images were observed over a period of $24 \mathrm{~h}$ post injection (representative images shown in Fig. 3a and b), which suggested there was no enhanced permeation and retention (EPR) effects by these two types of SPION in the tumor. In the case of N-pHLIP-SPION, decrease of MRI signal in the mouse liver were consistently found post injection. More importantly, time-dependent changes inside the tumor in MRI were observed over a period of $24 \mathrm{~h}$ post injection (Fig. 3c). In mouse number 1 , certain interior structure of tumor was revealed at $2 \mathrm{~h}$ post injection as compared to that prior injection, which then disappeared slowly at $4 \mathrm{~h}$ and even more at $24 \mathrm{~h}$. The tumor in mouse number 2 was found to have pronounced changes in the tumor perimeter in MRI at 2 and $4 \mathrm{~h}$, which then become blurry at $24 \mathrm{~h}$ post injection. Similar observation of changes in the tumor core of mouse number 3 was also found at less discernible level. Of note, these contrast changes were observed at 2-3 $\mathrm{mm}$ resolution based on the scale bar in the MR images (Fig. 3c). Because the mice in the MRI were taken out of the instrument at each time point, further quantitative analysis was not possible. However, the contrast differences shown in the MR images (Fig. 3) were sufficiently enough as a qualitative analysis. Thus, all these results implied that there was EPR effect in the tumor by the N-pHLIP-SPION in a time-dependent manner eliciting details of the tumor post injection (Fig. 3c). Therefore, selective targeting of liver tumor by SPION would not result in the loss of the tumor signals in the T2-weight MRI but provide additional dynamic details of tumor structures that would enhance the diagnosis of liver tumor at a small size below $8 \mathrm{~mm}$ size.

The selective liver tumor imaging in Fig. 3 might be attributed to the presence of N-terminus linked pHLIP peptide. The pH-induced membrane insertion mechanism by pHLIP is that the C-terminus of pHLIP was inserted across the cell membrane and located in the cytosol under acidic condition while the $\mathrm{N}$ terminus remained at the outside of cells. ${ }^{31-33}$ Therefore, the 
(a)

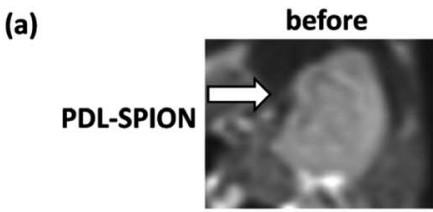

(b)

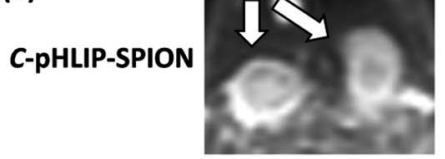

(c)
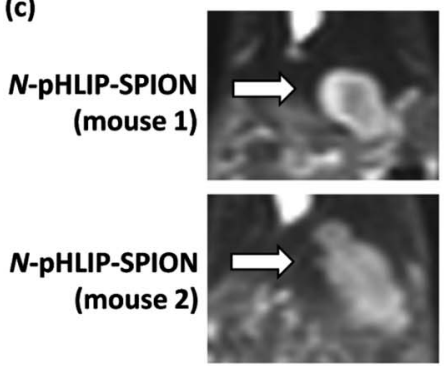

N-pHLIP-SPION

(mouse 3)

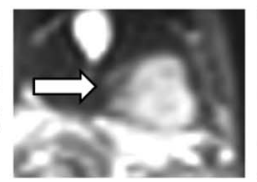

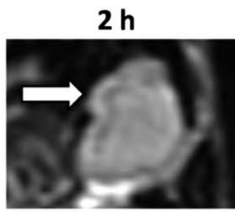
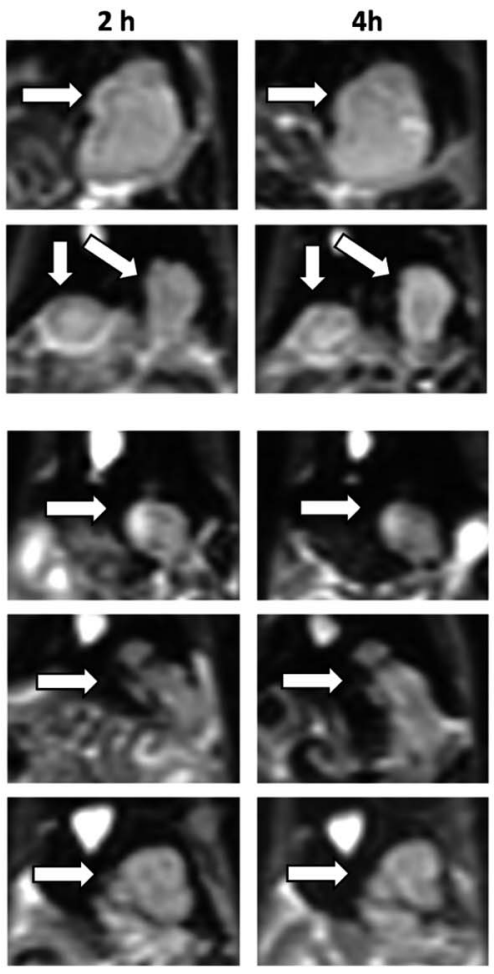

$24 \mathrm{~h}$
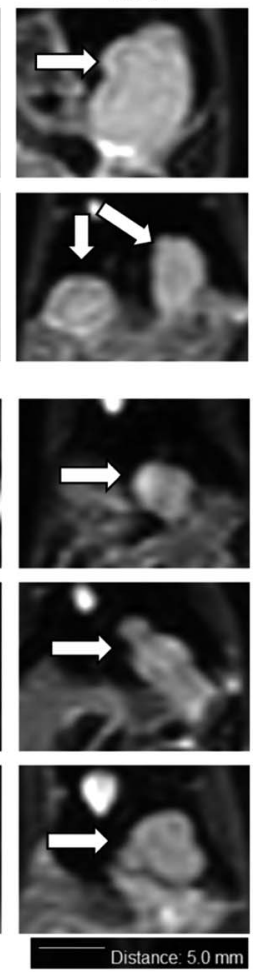

Fig. 3 Enhancement in the MRI analysis of orthotopic liver tumors with assembled SPION. Representative MR images in grayscale prior and post injections over $24 \mathrm{~h}$ with iv injection of (a) PDL-SPION, (b) C-pHLIP-SPION and (c) N-pHLIP-SPION at 5 mg Fe per kg body weight; tumors in MR images were indicated by white arrows with the scale bar of $5.0 \mathrm{~mm}$.

C-terminus linked SPION would require an endocytosis process to pull SPION into cytosol whereas the N-terminus linked SPION would possibly reside outside tumor cells. Although gold nanoparticles had been shown to be effectively delivered into tumor cells via the C-terminus linked pHLIP, ${ }^{34}$ no significant MRI contrast enhancement of tumors was observed with CpHLIP-SPION in our study, possibly due to the relatively large size of SPION and thus high membrane-cross energy required. In addition, the enhancement of tumor structure by N-pHLIPSPION was possibly due to the significantly overexpressed lactate dehydrogenase $\mathrm{A}$ in the tumor acidic cores. ${ }^{36}$

Besides the liver tumor, the MRI contrast enhancement in cirrhosis was also investigated with the assembled SPION. The purpose of the study was to evaluate whether the contrast enhancement in MRI by SPION was able to differentiate the fibrosis lesion from the tumor under the same condition. Ideally, an orthotopic liver tumor model with additional cirrhosis would validate the advantage of assembled SPION in the MRI diagnosis. Unfortunately, the mortality rate was so high that few mice survived for the investigation with MRI. It was found that all the cirrhosis mice exhibited ununiformed fiber-like pattern all across the liver prior SPIO injection similarly over $24 \mathrm{~h}$. Interestingly, the MRI signal of cirrhosis liver was markedly reduced post the injection of PDL-SPION, C-pHLIP-SPION or N-pHLIP-SPION (representative MRI at $4 \mathrm{~h}$ shown in Fig. 4). Only a small fraction of fiber-like pattern was barely observed post the SPION injection. Moreover, the contrast change in MRI clearly confirmed the enlarged liver organ in the cirrhosis with the maximum width $\times$ height about $20 \times 10 \mathrm{~mm}$ in Fig. 4 as compared with that of the liver in tumor model at $15 \times 7 \mathrm{~mm}$ size in Fig. 3 . The enhancement by SPIONs in cirrhosis had been attributed to that cirrhosis only resulted in partial dysfunctional Kupffer cells that were still capable to take up SPION. ${ }^{9-13}$

\subsection{Improved safety profile of N-pHLIP-SPION in cirrhosis model}

To ensure the potential safety of N-pHLIP-SPION as an MRI contrast agent for liver tumors, the toxicity profile of the assembled SPION was determined in cirrhosis model at the same injection dose. This was because although the poly-Llysine assembled SPION at $5 \mathrm{mg}$ Fe per $\mathrm{kg}$ injection dose had no toxicity impact in healthy mice, significant risk potential was discovered in cirrhosis model with disrupted liver function, lipid metabolism and iron level due to the iron overload post SPION injection, ${ }^{25}$ which indicated the safety profile in cirrhosis was a more better safety study model..$^{26}$ Thus, the serum liver function markers and lipid cholesterol levels including alkaline phosphatase (AKP), aspartate aminotransferase (AST), alanine aminotransferase (ALT), total cholesterol, high-density lipid cholesterol (HDL) and low-density lipid cholesterol (LDL) were monitored in cirrhosis mice over 15 days post the intravenous injection of assembled SPION. Analysis of liver functional serum markers is much more 
(a)

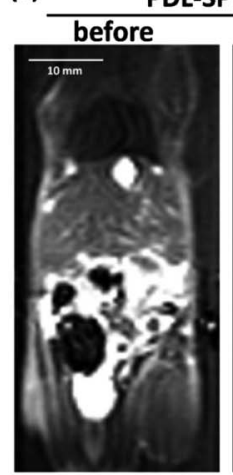

(b)

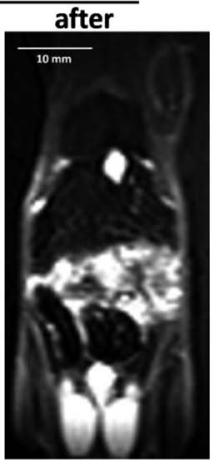

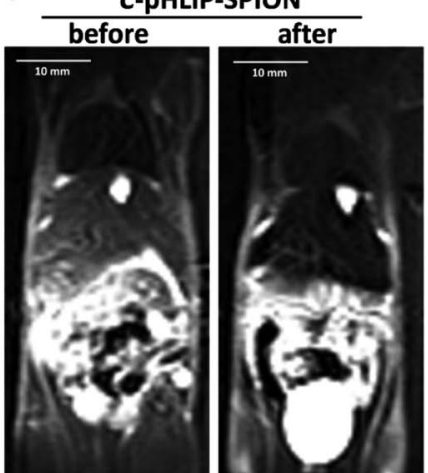

(c)

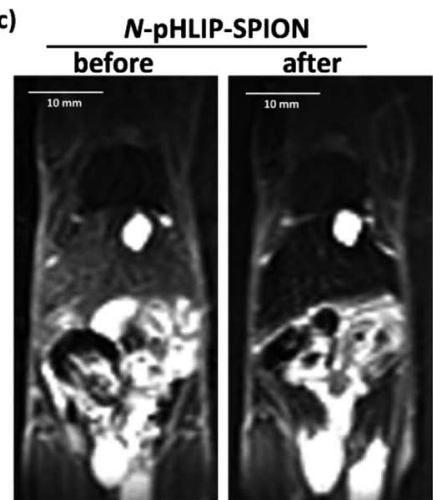

Fig. 4 Enhancement in the MRI of cirrhosis with assembled SPION. Representative MR images in grayscale prior and post injections of (a) PDLSPION, (b) C-pHLIP-SPION and (c) N-pHLIP-SPION at $5 \mathrm{mg}$ Fe per kg body weight at $4 \mathrm{~h}$.

accurate and indicative than the tissue pathological analysis, especial when the potential toxicity in cirrhosis is at the early stage. It was found that all three liver function markers increased at $24 \mathrm{~h}$ post the injection of the assembled SPION at $5 \mathrm{mg} \mathrm{Fe}$ per $\mathrm{kg}$ dose, possibly as a result of septic shock in response to the iv injection of SPION. ${ }^{25}$ The levels of these serum markers then decreased over 15 days with the ALT as the most decreased marker (Fig. 5a-c). While the serum cholesterol levels fluctuated a little bit over time after the initial increase post the injection (Fig. 5d-f). Among all six serum markers tested, only the total cholesterol and LDL levels had no difference among PDL-SPION, C-pHLIP-SPION and NpHLIP-SPION groups whereas the other four markers showed certain differences. All these results implied that impacts post the injection of assembled SPION were partial recoverable at various degree over 15 days in the cirrhosis model.

To fully assess whether the injection of assembled SPION had any potential toxicity impact on cirrhosis, we included two more control groups on day 15 as a comparison, namely, the cirrhosis group on day 15 with no SPIO injection as a negative control and the cirrhosis group on day 15 post injection of non-assembled SPIO as the positive control. It was found that cirrhosis without SPIO injection had an AKP level at $90 \mathrm{U} \mathrm{L}^{-1}$ on day 15 whereas the injection of non-assembled SPIO resulted in an increased level at $106 \mathrm{U} \mathrm{L}^{-1}$ even after 15 days (Fig. 6a). Similarly, the PDL-SPION injection induced a higher level at $111 \mathrm{U} \mathrm{L}^{-1}$ after 15 days whereas that of C-pHLIP-SPION was at $99 \mathrm{U} \mathrm{L}^{-1}$. Only the N-pHLIP-SPION group was the closest (a)

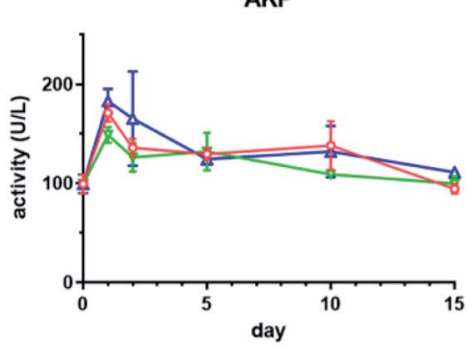

(d)

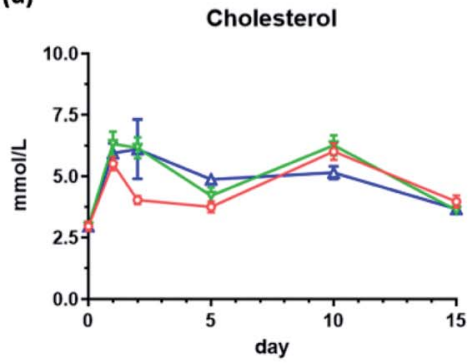

(b)

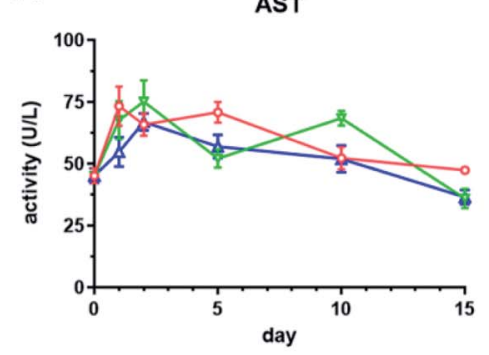

(e)

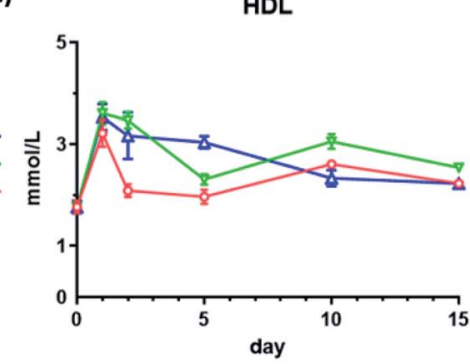

(c)

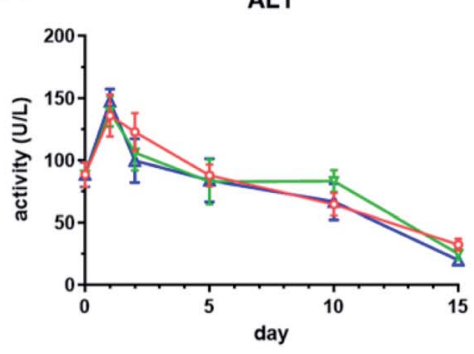

$\triangle$ PDL-SPION

$\rightarrow$ C-pHLIP-SPION

$\rightarrow$ N-pHLIP-SPION (f)

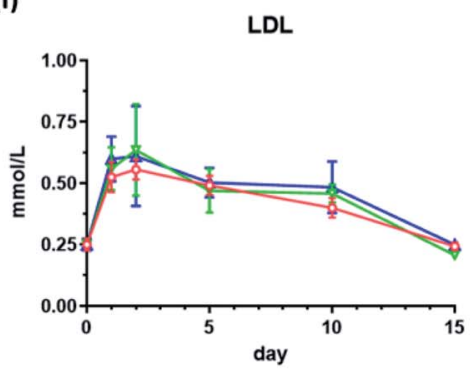

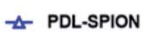

$\rightarrow$ C.PHLIP-SPION

$\rightarrow$ N-pHLIP-SPION

Fig. 5 Toxicity profiles of serum markers in cirrhosis model over 15 days post the intravenous injection of assembled SPION at 5.0 mg Fe per kg body weight. (a) AKP; (b) AST; (c) ALT; (d) total cholesterol; (e) HDL; (f) LDL; each data point represented the average of 5 mice per group with standard errors. 
(a)

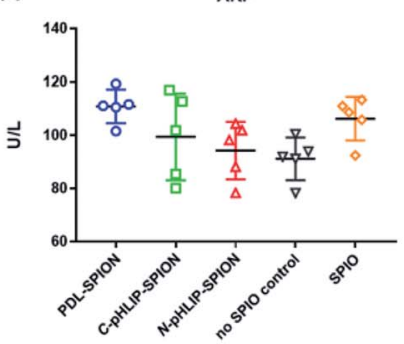

(e)

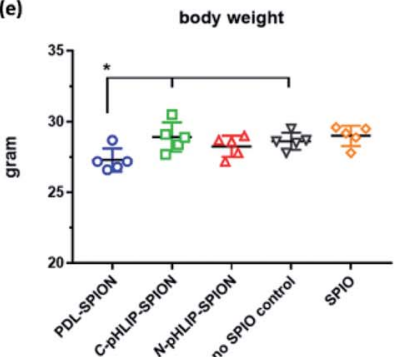

(b)

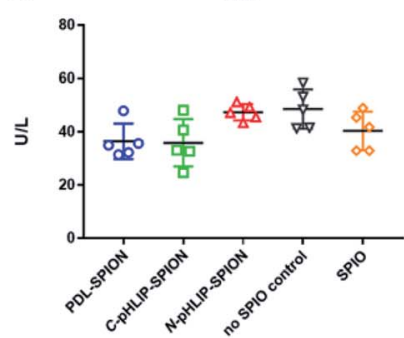

(f)

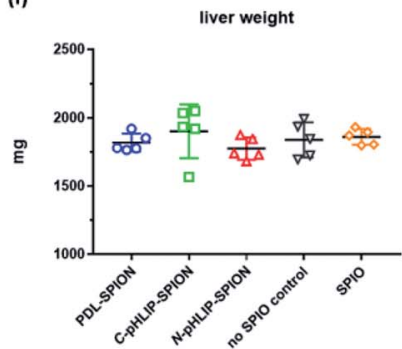

(c)

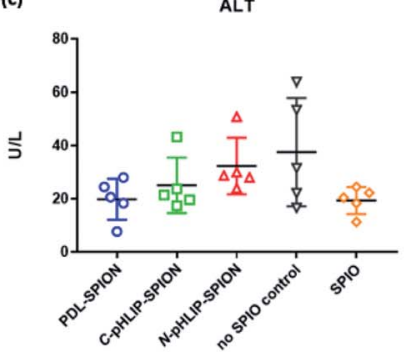

(g)

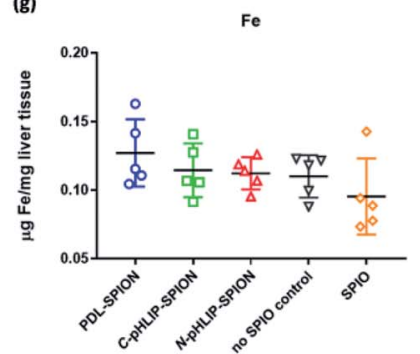

(d)

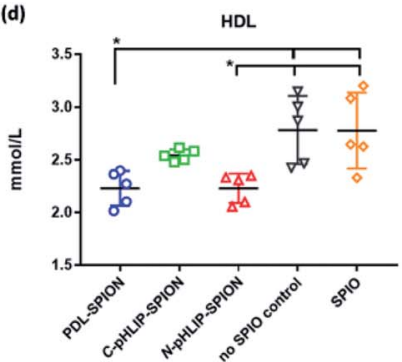

(h)

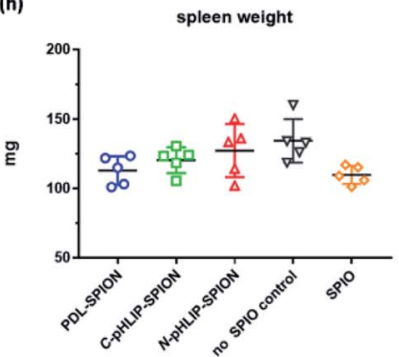

Fig. 6 Summary of serum markers and other safety parameters in cirrhosis model on day 15 post intravenous injection of assemble SPION or non-assembled SPIO at 5.0 mg Fe per kg body weight or with no SPIO injection. (a) AKP; (b) AST; (c) ALT; (d) HDL; (e) body weight; (f) whole liver weight; (g) iron level in liver tissue; (h) whole spleen weigh; each data point represented the average of 5 mice per group with standard errors ( $P$ $<0.01$ )

to no SPIO injection group at $94 \mathrm{U} \mathrm{L}^{-1}$. Similar trends were consistently observed in the AST and ALT levels, i.e., those of N-pHLIP-SPION were almost the same to those of no SPIO injection versus those of PDL-SPION and C-pHLIP-SPION similar to those of non-assembled SPIO injection (Fig. 6b and c). Unfortunately, due to the high variation of individual mouse, there was no statistically significant difference found. On the other hand, there was difference in the HDL level among the assembled SPION groups versus those nonassembled SPIO and no SPIO injection groups (Fig. 6d). Further comparison of the mouse body weight post injection indicated the PDL-SPION group had a significant one gram less body weight than other groups (Fig. 6e). It was found that the PDL-SPION group did not gain any body weight over 15 days post injection while other groups had 1-2 $\mathrm{g}$ weight increase because the mice in all groups had similar body weight on day 0 prior injection. On the other hand, the liver organ weights were all similar at approximated $1.8 \mathrm{~g}$ (Fig. 6f), characteristic of enlarged liver in cirrhosis as compare with the normally $1 \mathrm{~g}$ weight of the healthy mouse liver. ${ }^{25}$ In contrast, the iron level in the liver tissue was found to be different among these groups. The injection of non-assembled SPIO injection resulted in 10\% iron level loss in liver tissue whereas the PDL-SPION injection led to a $10 \%$ increase of iron level in the liver tissue after 15 days (Fig. 6g). Both of the pHLIP modified PDL-SPION had similar iron levels as that of no SPION injection group. Furthermore, decreased organ weight of the spleen was observed in the PDL-SPION and noneassembled SPIO group after 15 days in contrast to that of $\mathrm{N}$ pHLIP-SPION group that was closest to that of no SPIO injection group (Fig. 6h). Combined together, all these results indicated that N-pHLIP-SPION had an almost fully recoverable impact in the cirrhosis with similar liver function markers, iron level in liver tissue and spleen weight (except HDL levels) to that of no SPIO injection after 15 days post injection. On the other hand, non-assembled SPIO and PDL-SPION had high potential risk at the $5 \mathrm{mg}$ Fe per $\mathrm{kg}$ injection dose with increased AKP, decreased AST and ALT, altered iron level in liver tissue and decreased spleen organ weight.

Despite the relative high dose, the enhanced safety profile of N-pHLIP-SPION in cirrhosis demonstrated the feasibility for clinical applications. Our results implied that the enhanced safety profile was attribute to the modified PDL polymer assembly because both non-assemble SPIO and PDL-SPION resulted in disrupted safety markers as a high potential risk. On the other hand, the non-assembled SPIO, polylysine assembled SPION and pHLIP modified PDL assembled SPION were found to have low cytotoxicity in liver cells. ${ }^{30,35}$ Thus, it was the disrupted iron homeostasis due to iron overload by SPION in cirrhosis that was a safety concern. ${ }^{25,26}$ This was further supported by the reduced spleen weight observed after 15 days with SPIO and PDL-SPION injections. It has been shown that the macrophages in the liver and spleen were mainly responsible for the uptake of SPIO and that it would take over 30 days in spleen to slowly convert SPIO into iron in ferritin. ${ }^{37,38}$ In addition, our results showed that the Cterminus linked pHLIP SPION also had less toxicity than non-modified PDL-SPION. Thus, it was possible that pHLIP linked SPION could be metabolized more quickly in the liver and spleen due to the $\mathrm{pH}$ responsive nature, which was currently under investigation. 


\section{Conclusion}

We presented in this study that the N-terminus linked SPION as stable nanoclusters were a selective MRI contrast enhancement agent for liver tumor with significantly enhanced safety profile. N-pHLIP-SPION were capable to not only to "brighten up" the tumor through the reduction of MRI signal of the normal liver but also provided additional details of tumor in a timedependent manner post injection. In addition, the assembled SPION could be used for the MRI assessment of cirrhotic liver due to the uptake of SPION by partial dysfunctional Kupffer cells. Moreover, the injection of N-pHLIP-SPION had an almost fully recoverable impact in cirrhosis with a safety profile similar to that of cirrhosis group with no SPIO injection. In contrast, the PDL-assembled SPION and C-pHLIP-SPION showed non-tumor specific MRI contrast enhancement and potential safety risks in cirrhosis due to the iron overload by SPION. Therefore, targeting SPIONs in liver tumor would not result in the loss of the tumor signals in the T2-weight MRI but provide additional dynamic details of tumor structures that would enhance the diagnosis of liver tumor at a small size below $8 \mathrm{~mm}$ size. All these results implied the promising potential of N-pHLIPSPION as a selective MRI contrast agent for liver tumor diagnosis.

\section{Funding}

This work is supported by the National Natural Science Foundation of China (81671812, 81771819 and 81571734) and National Key Research and Development Plan of China (2017YFC0108803).

\section{Conflicts of interest}

The authors declare no conflicts of interest in this work.

\section{References}

1 Liver Cancer, Data source: GLOBOCAN 2018, International Agency for Research on Cancer, World Health Organization, http://gco.iarc.fr/today.

2 J. M. Llovet and J. Bruix, Hepatology, 2008, 48, 1312-1327.

3 S. Bota, F. Piscaglia, S. Marinelli, A. Pecorelli, E. Terzi and L. Bolondi, Liver Cancer, 2012, 1, 190-200.

4 M. Vivarelli, R. Montalti and A. Risaliti, World J. Gastroenterol., 2013, 19, 7316-7326.

5 H. J. Yoo, J. M. Lee, M. W. Lee, S. J. Kim, J. Y. Lee, J. K. Han and B. I. Choi, Invest. Radiol., 2008, 43, 538-546.

6 R. Golfieri, E. Marini, A. Bazzocchi, F. Fusco, F. Trevisani, C. Sama, G. Mazzella, S. Cavuto, F. Piscaglia and L. Bolondi, Radiol. Med., 2009, 114, 1239-1266.

7 J. Chung, J. S. Yu, D. J. Kim, J. J. Chung, J. H. Kim and K. W. Kim, Magn. Reson. Imaging, 2011, 29, 1235-1243.

8 L. M. Wu, J. R. Xu, Q. Lu, J. Hua, J. Chen and J. Hu, J. Gastroenterol. Hepatol., 2013, 28, 227-234.

9 L. P. Smits, B. F. Coolen, M. D. Panno, J. H. Runge, W. H. Nijhof, J. Verheij, M. Nieuwdorp, J. Stoker,
U. H. Beuers, A. J. Nederveen and E. S. Stroes, Radiology, 2016, 278, 782-791.

10 S. Maurea, P. P. Mainenti, A. Tambasco, M. Imbriaco, C. Mollica, E. Laccetti, L. Camera, R. Liuzzi and M. Salvatore, Quant. Imaging Med. Surg., 2014, 4, 181-189.

11 A. Nishie, Y. Asayama, K. Ishigami, T. Tajima, D. Kakihara,

T. Nakayama, Y. Takayama, D. Okamoto, A. Taketomi,

K. Shirabe, N. Fujita, M. Obara, K. Yoshimitsu and

H. Honda, J. Magn. Reson. Imaging, 2012, 36, 664-671.

12 T. Tonan, K. Fujimoto, A. Qayyum, Y. Morita, O. Nakashima, N. Ono, A. Kawahara, M. Kage, N. Hayabuchi and T. Ueno, J. Gastroenterol. Hepatol., 2012, 27, 789-796.

13 H. Cheong, S. S. Lee, J. S. Lee, J. Kim, S. W. Kim and W. J. Lee, J. Magn. Reson. Imaging, 2015, 41, 1218-1227.

14 J. E. Rosen, L. Chan, D. B. Shieh and F. X. Gu, Nanomedicine, 2012, 8, 275-290.

15 R. A. Revia and M. Zhang, Mater. Today, 2016, 19, 157-168. 16 L. S. Arias, J. P. Pessan, A. P. M. Vieira, T. M. T. Lima, A. C. B. Delbem and D. R. Monteiro, Antibiotics, 2018, 7, E46. 17 D. Chang, M. Lim, J. A. C. M. Goos, R. Qiao, Y. Y. Ng, F. M. Mansfeld, M. Jackson, T. P. Davis and M. Kavallaris, Front. Pharmacol., 2018, 9, 831.

18 J. Hu, Y. Qian, X. Wang, T. Liu and S. Liu, Langmuir, 2012, 28, 2073-2082.

19 K. Zhu, Z. Deng, G. Liu, J. Hu and S. Liu, Macromolecules, 2017, 50, 1113-1125.

20 V. Mirshafiee, B. Sun, C. H. Chang, Y. P. Liao, W. Jiang, J. Jiang, X. Liu, X. Wang, T. Xia and A. E. Nel, ACS Nano, 2018, 12, 3836-3852.

21 V. Mirshafiee, W. Jiang, B. Sun, X. Wang and T. Xia, Mol. Ther., 2017, 25, 1522-1530.

22 N. Fujita, H. Miyachi, H. Tanaka, M. Takeo, N. Nakagawa, Y. Kobayashi, M. Iwasa, S. Watanabe and Y. Takei, Cancer Epidemiol., Biomarkers Prev., 2009, 18, 424-432.

23 U. Ahmed, P. S. Latham and P. S. Oates, World J. Gastroenterol., 2012, 18, 4651-4658.

24 M. Lunova, C. Goehring, D. Kuscuoglu, K. Mueller, Y. Chen, P. Walther, J. C. Deschemin, S. Vaulont, J. Haybaeck, C. Lackner, C. Trautwein and P. Strnad, J. Hepatol., 2014, 61, 633-641.

25 Y. Wei, M. Zhao, F. Yang, Y. Mao, H. Xie and Q. Zhou, Sci. Rep., 2016, 6, 29110.

26 Q. Zhou and Y. Wei, Chem. Res. Toxicol., 2017, 30, 73-80.

27 Z. Bakhtiary, A. A. Saei, M. J. Hajipour, M. Raoufi, O. Vermesh and M. Mahmoudi, Nanomedicine, 2016, 12, 287-307.

28 N. Schleich, C. Po, D. Jacobs, B. Ucakar, B. Gallez, F. Danhier and V. Préat, J. Controlled Release, 2014, 194, 82-91.

29 C. Zhang, H. Liu, Y. Cui, Z. Zhang, T. Xu, Y. Zhang and D. Wang, Int. J. Nanomed., 2016, 11, 1097-1108.

30 Y. Wei, R. Liao, A. A. Mahmood, H. Xu and Q. Zhou, Acta Biomater., 2017, 55, 194-203.

31 D. Weerakkody, A. Moshnikova, M. S. Thakur, V. Moshnikova, J. Daniels, D. M. Engelmanm, O. A. Andreev and Y. K. Reshetnyak, Proc. Natl. Acad. Sci. U. S. A., 2013, 110, 5834-5839. 
32 O. A. Andreev, A. D. Dupuy, M. Segala, S. Sandugu, D. A. Serra, C. O. Chichester, D. M. Engelman and Y. K. Reshetnyak, Proc. Natl. Acad. Sci. U. S. A., 2007, 104, 7893-7898.

33 O. A. Andreev, D. M. Engelman and Y. K. Reshetnyak, Mol. Membr. Biol., 2010, 27, 341-352.

34 A. Davies, D. J. Lewis, S. P. Watson, S. G. Thomas and Z. Pikramenou, Proc. Natl. Acad. Sci. U. S. A., 2012, 109, 1862-1867.
35 Y. Wei, R. Liao, H. Liu, H. Li, H. Xu and Q. Zhou, J. Biomed. Nanotechnol., 2015, 11, 854-864.

36 R. C. Adochite, A. Moshnikova, S. D. Carlin, R. A. Guerrieri, O. A. Andreev, J. S. Lewis and Y. K. Reshetnyak, Mol. Pharm., 2014, 11, 2896-2905.

37 H. L. Ma, Y. F. Xu, X. R. Qi, Y. Maitani and T. Nagai, Int. J. Pharm., 2008, 354, 217-226.

38 J. D. López-Castro, A. V. Maraloiu, J. J. Delgado, J. J. Calvino, M. G. Blanchin, N. Gálvez and J. M. Domínguez-Vera, Nanoscale, 2011, 3, 4597-4599. 